Published in final edited form as:

Curr Protoc Nucleic Acid Chem. 2001 May ; 0 7: Unit-7.8. doi:10.1002/0471142700.nc0708s04.

\title{
Molecular Modeling of Nucleic Acid Structure: Energy and Sampling
}

\author{
Christina Bergonzo, Rodrigo Galindo-Murillo, and Thomas E. Cheatham III
}

\begin{abstract}
An overview of computer simulation techniques as applied to nucleic acid systems is presented. This unit discusses methods used to treat the energy and to sample representative configurations. Emphasis is placed on molecular mechanics and empirical force fields.
\end{abstract}

\section{Subject Group \\ Nucleic Acid Chemistry; Nucleic Acid Structure and Folding; Structural Analysis of Biomolecules; Experimental Determination of Structure; Molecular Modeling; Molecular Dynamics; Force Fields}

\section{THE ENERGY REPRESENTATION}

To aid a modeler in judging the reliability of a given model structure, it is useful to include some representation of the energy. The presumption is that structures with lower energiessuch as those with less steric overlap, less distortion, and more favorable interactions-will

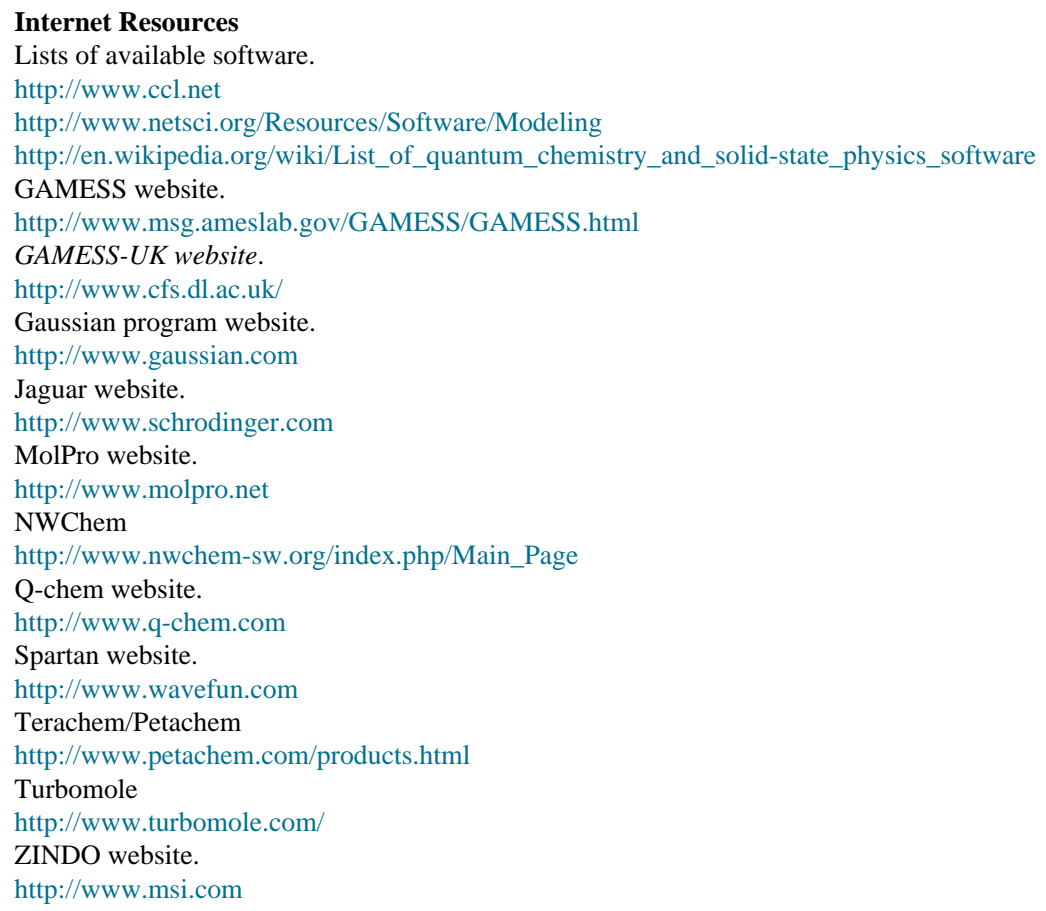


be more representative. As discussed (UNIT 7.5), adding a representation of the energy to a physically manipulatable model is difficult; however, some implementation of the energy for a given molecular model can be easily programmed on a computer. In common use, the level of theory applied spans the range from complex and highly accurate ab initio quantum mechanical $(\mathrm{QM})$ to simpler empirical molecular mechanical energy treatments, to coarse grain models for extremely large systems. As the accuracy of the energy increases, so does the computational cost of performing the calculation. The increase in computational cost from a coarse grained representation to ab initio QM is tremendous and limits the level of theory that may be applied to a given system. For calculating the energy of a single model structure, very accurate methods can be applied; however, if one is interested in dynamics or investigating the energy of many configurations of a given model, less accurate energy representations are generally necessary.

\section{Quantum Mechanical (QM) Treatments}

To provide an accurate and complete theoretical description of the energy (as a function of the atomic and electronic configuration or structure) of a molecular system, ab initio QM treatments can be applied. Standard codes for performing QM calculations include Gaussian, Jaguar, Q-Chem, GAMESS, MOLPRO, NWCHEM, Spartan, Turbomole, TeraChem, etc (see Internet Resources). Performing calculations with these programs is fairly straightforward, even for those without a broad theoretical background in the methods. For reviews of QM methods, see the books by McQuarrie and Simon (1997), Szabo and Ostlund (1989), or Levine (1991, $6^{\text {th }}$ ed. 2008). A serious drawback of these methods is that they are extremely computationally demanding, which typically limits highly accurate QM calculations to small model systems (<500atoms). Recent improvements in the methods, coupled with the availability of greater computational power, have led to more involved and highly accurate QM calculations. Accuracy is improved with the use of larger and higher level basis sets and with the inclusion of electron correlation. The use of density functional methods allows for the investigation of larger systems (and implicitly includes some electron correlation); however, even with these improvements, tractable model systems are still size limited to individual base pairs or stacked nucleotides, although calculations are beginning to emerge on larger systems such as tetranucleotides or two or more quartets from a G-DNA quadruplex (Cheatham, Sponer, unpublished). The likely largest use of QM methods in nucleic acid modeling is in the development, evaluation, and critique of empirical potential functions (as are introduced later in this section).

Two important considerations in QM representation of nucleic acids are solvation and stacking. The effect of the solvent, if included at all, can only be included implicitly (via a mean field or continuum treatment) or through the inclusion of a very small number of explicit waters around the molecule in the QM calculation. A polarizable continuum solvation model (PCM) is available for Hartree Fock (HF) and density functional theory (DFT) levels of theory in many QM packages. PCM describes the continuum as a polarizable dielectric (Cances et al., 1997). An alternative is the COSMO approach which uses a scaled conductor approximation in deriving the charges of the continuum (Klamt and Schuurmann, 1993). A review of quantum mechanical continuum solvation methods can be found in Tomasi et al., 2005. A known issue of many DFT methods are that they tend to 
underestimate dispersion attraction interactions and therefore underestimate base pair stacking. As inclusion of noncovalent dispersion forces is critical in modeling stacking in conjugated $\pi$ systems (Grimme 2011), empirical corrections can be added to correct dispersion, for example by using the Truhlar family of functionals (Zhou and Truhlar, 2008) or the PBE hybrid functionals. A good benchmark demonstrating how these methods correctly account for dispersion interactions is available from Risthaus and Grimme (2013).

Prior to using QM methods to investigate nucleic acid models, it would be wise to consult the detailed literature for similar examples. Some recent examples of QM treatments of nucleic acids include the investigation of base-pairing energetics, base stacking, the interaction of metal ions with base pairs, and non-canonical hydrogen bonding(for reviews see Hobza and Sponer, 1999, Sponer et al., 2008, Sponer et al., 2010, and Sponer et al., 2012). QM calculations have also been applied directly in macromolecular modeling applications via combined QM/MM potentials. Most often the QM/MM methods are applied to model catalysis by ribozymes (Banas et al., 2009, Mlynsky et al., 2011, Sgrignani and Magistrato, 2012).

As mentioned in UNIT 7.5, although the QM treatments have the potential to provide a very high level of accuracy, this level of accuracy is not always required, and faster, less accurate techniques may be appropriate; however, the commonly applied approximations come at a cost. Without a QM treatment, it is generally not possible to accurately represent processes that involve chemical changes (i.e., chemical reaction, bond breaking, or bond forming), excited states (i.e., electronic transitions), or electron transfer. In practice this is no longer a major limitation, since with nucleic acids the investigator is most often simply interested in the structure, dynamics, and relative importance of a given model, and when bond breaking or forming is desired a combined QM/MM method can be used. In practice, this is often done in the middle ground between pure ab initio QM techniques and the faster empirical potentials (discussed in the next section; see Molecular Mechanics: Empirical Potential Energy Functions), specifically semi-empirical treatments. These apply a quantum mechanical formalism where significant approximations are applied in the calculation to decrease the computational cost, while accuracy lost through the approximations is offset by the addition of empirical parameters (Lee et al., 2008, Nam et al., 2008, Yang et al., 2008). The semi-empirical methods allow investigation of 2500 atoms for geometry optimization or $\sim 100$ atoms for dynamics in the QM regions. Their use in biomolecular simulations has been limited in part due to the difficulty in properly representing hydrogen-bonded systems, although newer methods such as SCC-DFTB show promise. Semi-empirical parameterizations for biomolecular systems, methods for treating large systems (Thiel, 1997), and semi-empirical codes designed to run on massively parallel computers (Dixon and Merz, 1997) have been developed, but are generally known to be unsuitable for noncovalent interactions, and in some cases do not outperform empirical potentials (Hobza et al. 1998). Standard semi-empirical codes include NDDO, MOPAC, MNDO4, AMSOL, Argus, and ZINDO (see Internet Resources) among others, and utilize various parameterizations including AM1, PM3, and MNDO. Alternatives include RM1, PDDG/ PM3, PDDG/MNDO, and PM6 semi-empirical parameterizations. The RM1 reparameterization of AM1 used a reference set of organic and biological molecules to 
improve quantitative accuracy while preserving AM1's decreased computational cost (Rocha et al., 2006). Good behavior is also seen by introducing functional group information via corrections to the core repulsion function as applied in the PDDG/PM3and PDDG/ MNDO models (Repasky et al., 2002). More recent parameterizations, such as the PM6 method, can account for hydrogen bonding explicitly, and therefore these return more accurate results (Rezac et al., 2009) with further improvement using various corrections (Korth 2010; Korth et al. 2010). Reviews on semi-empirical methods discuss the codes and parameterizations in greater detail (Stewart, 1990; Zerner, 1991; Riley et al., 2010).

Despite the higher levels of accuracy possible with the quantum mechanical and semiempirical treatments, the most commonly applied treatment of energy uses simplified molecular mechanical (MM) potential energy functions. Given an appropriate parameterization, these MM potential functions are able to accurately reproduce nucleic acid structure at the atomic level in a very computationally efficient manner. This allows rapid evaluation of not only energy, but forces (or the first derivative of the energies with respect to the positions), thereby allowing analysis of many configurations in a reasonable time frame.

Before leaving this discussion of ab initio and semi-empirical methods, it is important to mention that there has been significant progress in the development of hybrid QM/MM methods, as already briefly mentioned. These treat part of the system quantum mechanically (the part undergoing chemical change) and the remainder with a significantly faster molecular mechanical (empirical) potential. The hybrid QM/MM treatments allow representation of larger systems (such as ribozymes) with explicit representation of the environment, while still treating the region of chemical interest (such as the active site) quantum mechanically. Drawbacks of these methods are that they are extremely computationally demanding (depending in large part on the size of the QM region) and that there are a number of system-specific research questions, such as how best to merge the QM and MM regions, how best to decide what part of the system should be represented quantum mechanically, and what level of treatment to apply in the QM region (i.e., ab initio versus specifically parameterized semi-empirical) or MM regions (Field et al., 1990; Stanton et al., 1995; Gao, 1996; Cummins and Gready, 1997; Chatfield et al., 1998). Though most of the early QM/MM applications have been limited to enzyme systems or small molecules in solution, applications towards nucleic acid systems beyond those already mentioned modelribozyme catalysis (Lahiri and Nilsson, 1997), nucleotidyl transfer reactions in the polymerase active site (Lior-Hoffmann, 2012), and free radical induced DNA damage (Abolfath 2012), among many others (for a review see Sann and Thiel, 2009, Rokob et al. 2012). Code for performing $\mathrm{QM} / \mathrm{MM}$ is in many of the standard molecular dynamics programs, including AMBER (Case et al., 2005), CHARMM (Brooks et al., 2009), and GROMACS (Hess et al., 2008), and also in many of the standard QM codes.

\section{Molecular Mechanics: Empirical Potential Energy Functions}

The most commonly applied method for describing energy is to use an empirically derived or molecular mechanics (MM) potential function. This involves the application of a simplified potential function that has been parameterized to properly model the structures of 
interest. The specific parameterization, or force field, needs to represent not only the intramolecular interactions (based on the covalent structure of the molecule) but the intermolecular interactions between all the atoms and molecules. Most of the commonly applied empirical derived force fields describe the intermolecular interactions in a similar manner. The complete and true atomic energy representation, $U\left(r_{1}, r_{2}, \ldots, r_{N}\right)$, involves the interactions of all $N$ atoms (at positions $r_{l}$ to $r_{N}$ ) in the system. Following Allen and Tildesley (1987), this energy representation can be decomposed into a sum of pairs, triples, quadruples, and higher interactions between atom centers:

$$
U\left(r_{1}, r_{2}, \ldots, r_{N}\right)=\sum_{i=1}^{N} U\left(r_{i}\right)+\sum_{i=1}^{N} \sum_{j>1}^{N} U\left(r_{i}, r_{j}\right)+\sum_{i=1}^{N} \sum_{j>i}^{N} \sum_{k>j>i}^{N} U\left(r_{i}, r_{j}, r_{k}\right)+\ldots \quad \text { Equation }
$$

Approximations are then applied to simplify the representation. Given that the individual interactions for the terms from the quadrupolar interaction and higher-order interactions are usually very small, these are often neglected. The first-order term, $U\left(r_{i}\right)$, involves interactions with external fields and, therefore, is also generally not included. This leaves the dominant terms, specifically the pair interactions (a function of the distance between the atoms; $r_{i j}$ ) and the three-body (atom-dipolar) interactions. The three-body interactions are less often included explicitly in biomolecular simulation at present due to the increased cost of calculation and the nonadditivity of the energy; however, these interactions are not completely neglected since their effect is implicitly included in the molecular mechanical force field during the explicit parameterization of the pairwise interactions. To summarize, the most commonly applied empirical force fields use an additive pairwise potential (with the nonadditivity and three-body terms omitted).

\section{Intramolecular Interactions}

The intramolecular interactions describe the covalent structure of the molecule. This includes the bonds, angles, dihedrals, and overall connectivity and flexibility of the model. A reasonable MM representation can be obtained either with full atomic or internal coordinate representations. Atomic representations imply that each atom center is allowed to move independently and explicit bond, angle, and dihedral terms are added to represent the connectivity. Two common representations are applied: all-atom force fields treat each atom as independent, whereas united-atom force fields fold multiple atomic centers into a single particle, such as treating a methylene group $\left(-\mathrm{CH}_{2}-\right)$ as a single center.

Internal coordinate representations attempt to represent the inherent motions and flexibility of the system via rotations about naturally rigid groups of atoms. These can be useful since there are effectively fewer degrees of freedom, with flexibility only included where necessary to represent the natural motion of the molecule. For example, with nucleic acids the bases can often be treated as rigid units, as can most of the backbone (except for $\varepsilon$ and $\zeta$ ), with rotations and translations set up to represent deviations from a common helical axis system, such as rotations involving inclination, tip and twist, and translations (e.g., $x$ - and $y$ displacement from the helical axis and rise between base pairs). To include the flexibility of the sugar pucker, the bonds at the $\mathrm{C} 4^{\prime}-\mathrm{O} 4^{\prime}$ atoms can be broken, leading to two torsions and 
three angles to describe the ring. This internal coordinate representation is used in the program JUMNA (junction minimization of nucleic acids; Lavery et al., 1995, Harvey et al. 2002)or with the Flex force field (Flatters et al., 1997). Alternative treatments may use different rotations (e.g., twist, roll, and tilt; Gorin et al., 1990) and more complicated treatments that allow more complicated pucker and backbone changes necessitating analytical closure procedures for the rings (Nesterova et al., 1997). Another alternative, applied with some protein force fields such as the UNRES force field (Maisuradze et al., 2010), is to keep all or some of the bonds and angles fixed while allowing free rotation around all the torsions. For a more detailed review of coarse-grained treatments of nucleic acids, see the cited reviews (de Pablo, 2011, Flores, 2011, Takada, 2012).

Ideally, a good internal coordinate representation will attempt to minimize the number of degrees of freedom while still retaining good structure and dynamics. Reduction of the number of degrees of freedom is desirable since this has clear benefits when trying to sample the possible conformations (as will become more apparent later). Although the internal coordinate treatments lead to fewer degrees of freedom, using this kind of representation in molecular dynamics simulations is difficult because accurate treatment requires inverting the moment of inertia tensor (or mass matrix) or the application of computationally demanding holonomic constraints (that maintain the fixed structure within rigid groups and properly equalize or propagate the forces). In all-atom or united-atom treatments, because each atom center is free to move, the mass matrix is diagonal and thus, trivially inverted. Even though there are approximations that effectively treat the moment of inertia tensor when it is not diagonal with order $N$ rather than $N^{3}$, accuracy is lost. Based on these efficiency issues, internal coordinate treatments are generally only used with minimization or Monte Carlo simulation (as described more fully in the next section). A further potential difficulty with the internal coordinate representation is that it requires specification of the rigid units; if the unit is rigid, it cannot distort structurally, in contrast to what might be the expected behavior under certain conditions. Thus, care has to be taken not to rigidify a part of the molecule that may not be rigid in practice. Moreover, rigid rotation about a given bond effectively leads to higher rotational barriers since there is no coupling to other modes (i.e., the bonds or angles cannot open up to facilitate rotation). For example, the gauche, gauche rotational barrier for butane is roughly twice as large when the bonds and angles are held rigid. Of course, this artifact is not a problem in practice since the force fields are parameterized to compensate; this just points out that it is not possible to directly mix intramolecular force fields designed for use with internal coordinates with those designed for use in all-atom treatments.

The alternative to using an internal coordinate treatment is to not treat any of the internal coordinates as rigid, so that each atom is free to move. In this case, to represent the covalent structure and intramolecular energetics, explicit energetic representations for the bonds, angles, and dihedrals need to be added. In the simplest form, this is typically done with harmonic potentials to maintain bond lengths and angles, and Fourier terms for the torsion angles to represent rotation about bonds (Figure 7.8.1). Either harmonic or Fourier terms are also commonly added to maintain planarity or to prevent rotation about double bonds. 
Higher-order terms can also be added as necessary for more detailed representation. A common form for the intramolecular part of the mechanical potential function is as follows:

$$
U_{\text {intramolecular }}=\sum_{\text {bonds }} k_{b}\left(r-r_{e q}\right)^{2}+\sum_{\text {angles }} k_{\theta}\left(\theta-\theta_{e q}\right)^{2}+\sum_{\text {dihedrals }} \sum_{\eta} \frac{V_{\eta}}{2}[1+\cos (\eta \varphi-\gamma)]+\ldots \quad \text { Equation }
$$

The parameterization, or force field, refers to the specific equilibrium geometry values for the various terms (such the equilibrium bond length, $r_{\mathrm{eq}}$ ) and the force constants representing the energy (or vibrational frequency, $k_{\mathrm{b}}$ ) of distortion away from the equilibrium geometry. The parameterization is typically performed on molecular fragments with the implicit assumption that the force field parameters are transferable to other fragments. In other words, a carbon-carbon bond in propane is the same as a carbon-carbon bond in pentane. Bond lengths and bond angles are determined via experiment (crystallography or other spectroscopic techniques) with force constants for the vibration inferred from microwave, infrared (IR), other spectroscopic data, and sometimes high level QM data. In the parameterization, the least well-determined parameters (based on experimental information) are the dihedral terms, since these terms effectively include not only the equilibrium torsion value but 1-4 non-bonded atom interactions and other implicit interactions which are explicitly omitted. Given this, the dihedral part of the force field is usually the last part to be parameterized (based on QM and empirical data) and modification of these parameters can be used to fix up deficiencies from the other intramolecular and intermolecular interactions.

More complex molecular mechanical representations are also possible, such as those including cubic and quartic terms for bond stretching, cubic angle terms for an harmonic bending, bond-torsion, angle-torsion, bend-bend, and other terms. Although these higherorder terms can aid in parameterization efforts to better represent vibrational spectra, structure, and heats of formation in a diverse set of molecules (including strained molecules), these are not typically included in biomolecular force fields. This is because biomolecular force fields are primarily parameterized to represent structure and secondarily relative conformational energetics in as simple and transferable a means as possible. An adequate representation is obtained without the added complexity, as will become apparent. For more information about the more strongly parameterized class 2 and class 3 force fields that include the more complicated intramolecular energy representation, see discussions of the following force fields: MMFF94 (Halgren, 1996), MM3 and MM4 (Allinger et al., 1989, 1996), and QMFF (Maple et al., 1994).

\section{Intermolecular Interactions}

The standard form for the intermolecular pair energy involves a Lennard-Jones potential to represent the electron cloud repulsion $\left(r_{i j}{ }^{-}{ }^{12}\right)$, dispersion attraction $\left(r_{i j}{ }^{-}{ }^{-6}\right)$ interactions, and a Coulombic term (with atom point charges $q_{i}$ and $q_{j}$, and dielectric constant $\varepsilon$ ) representing the electrostatic interactions between all the atom pairs (where $r_{i j}$ is the distance between atoms $i$ and $j$ ). 


$$
U_{\text {intermolecular }}=\frac{A_{i j}}{r_{i j}^{12}}-\frac{B_{i j}}{r_{i j}^{6}}+\frac{q_{i} q_{j}}{\varepsilon r_{i j}} \quad \text { Equation 7.8.3 }
$$

Note that the dielectric constant $(\varepsilon)$ is shown in a simplified form that implicitly includes the $4 \pi \varepsilon_{0}$ leading factor, where $\varepsilon_{0}$ is the permittivity of free space. Also note that selfinteractions (1-1), interactions between bonded atoms (1-2), and interactions between the atoms involved in angles (1-3) are most often omitted, since their interaction has already been included in the intramolecular part of the force field. Interactions between terminal atoms involved in a dihedral angle (1-4 interactions) are sometimes scaled since their interactions are partially embedded in the associated bond and angles. Polarization effects are also often included implicitly in the parameterization, although explicit polarization (as is discussed later) can be included at additional cost. The specific parameters $\left(A_{i j}, B_{i j}, q_{i}, q_{j}\right)$ in large part determine the reliability of the intermolecular potential. There are many philosophies regarding how to "best" derive these parameters, ranging from total reliance on high-level QM treatments of fragments to parameterization based entirely on empirical data, or some combination of each. Additionally, the Lennard-Jones parameters for particular atom types have been derived from simulations of neat liquids (Jorgensen et al., 1996). As with the bond, angle, and dihedral parameters, there is an implicit assumption that (in general) the Lennard-Jones parameters are transferable. It should be noted that the parameters $A_{i j}$ and $B_{i j}$ for the Lennard-Jones part of the equation above represent mixed van der Waal parameters for atoms $i$ and $j$. Since the literature is sometimes confusing with presentation of the mixed parameters as $A_{i j}$ and $B_{i j}$ (with or without pre-exponentiation) or in terms of $\varepsilon$ (the potential well depth)and either $r^{*}$ (the minimum of the potential well in $\AA$ ) or $\sigma$ (the zero of the potential in $\AA$ ), and since this is further complicated by application of different combining rules, it is worth a brief digression. The two forms of the van der Waals energy in terms of $\varepsilon, r^{*}$ and $\sigma$ are as follows:

$$
E_{\mathrm{LJ}}=4 \varepsilon\left[\left(\frac{\sigma}{r_{i j}}\right)^{12}-\left(\frac{\sigma}{r_{i j}}\right)^{6}\right]=\varepsilon\left[\left(\frac{r^{*}}{r_{i j}}\right)^{12}-2\left(\frac{r^{*}}{r_{i j}}\right)^{6}\right] \quad \text { Equation 7.8.4 }
$$

This implies that:

$$
r^{*}=\sigma \sqrt[6]{2} \quad \text { Equation 7.8.5 }
$$

Given an $r^{*}$ value (representing the van der Waal radius) for two atoms $i$ and $j$ that are not the same, it is necessary to define a mixed van der Waal radius. There are two methods in common usage, arithmetic or Lorentz-Bertholet combining rules (as applied in AMBER and CHARMM) where:

$$
r_{i j}^{*}=\frac{r_{i}^{*}+r_{j}^{*}}{2} \quad \text { Equation 7.8.6 }
$$

and geometric mean combining rules (as applied in GROMOS and OPLS-AA) where: 


$$
r_{i j}^{*}=\sqrt{r_{i}^{*} r_{j}^{*}} \quad \text { Equation 7.8.7 }
$$

In both cases, a geometric mean is used for the well depth. These differences again point to the critical need to be careful when trying to adapt force fields applied in one program for use in another or, alternatively, in mixing parameters from different force fields.

Other forms for the intermolecular potential can also be employed to represent the intermolecular interactions, such as replacing the repulsive (12) potential by an exponential, as in MM2 (Allinger, 1977), or replacing the Lennard-Jones potential by a buffered 7-14 potential for the van der Waal interactions and shifting the electrostatics to prevent infinite attractive electrostatics from dominating the finite van der Waals interactions at short range, as employed in MMFF94 (Halgren, 1996). Although these more complicated functional forms arguably work much better for the varied small molecules of interest to pharmaceutical companies, these force fields have not seen significant use in biomolecular simulation applied to nucleic acids.

For the electrostatic interactions, most MM methods assume fixed atomic point charges. Polarization is only included implicitly via the construction of point charge values that lead to effectively larger dipoles, for example with the AMBER force fields where electrostatic potential fits of charges to 6-31G* optimized QM geometries are applied since this basis set tends to overestimate the dipoles. The point charges are one part of the MM model that is not very transferable since a given atomic charge depends critically on its environment; therefore, charges are typically calculated for individual fragments (such as each individual nucleic acid or amino acid) rather than for specific atom types. With the lack of explicit polarization, a major limitation of the additive pairwise force fields is in the treatment of transition metals or multivalent ions (which may not be treated properly with standard additive empirical force fields); in this case, accurate treatment may require the inclusion of explicit polarization effects or even some QM treatment.

Polarization effects can be included in the MM potential energy function. Methods which address polarizability include fluctuating charge, Drude oscillator, inducible dipoles, electronic polarization based on QM treatments (Biancardi et al, 2013), and even methods which mix polarizable treatments with continuum solvation (Tan et al, 2008). Currently available polarizable force fields for nucleic acids tend to use either inducible dipoles (Wang et al, 2011), sometimes with higher order multipoles included as with the AMOEBA force fields (Ponder et al, 2010)—although a nucleic acid force field for AMOEBA has not yet emerged,- - or Drude oscillator approaches (Baker 2011). Arguably, with the exception of the Drude oscillator force fields in CHARMM, at present, none of the polarizable force fields for nucleic acids perform better than the best available additive force fields. However, for completeness, we introduce the formalism behind inducible dipoles. For deeper understanding of related methods, consult the published literature. 
The formalism for including inducible dipoles on each center $\left(\mu_{i}\right)$ reacting to the electrostatic field $\left(E_{i}{ }^{0}\right)$ on center $i$ arising from all other fixed charges and representing the polarization energy $\left(U_{\text {pol }}\right)$ is as follows:

$$
U_{\mathrm{pol}}=-\frac{1}{2} \sum_{i} \mu_{i} E_{i}^{0} \quad \text { Equation 7.8.8 }
$$

This is evaluated self-consistently, solving for the inducible dipole based on the polarizability of atom $i\left(\mathrm{a}_{i}\right)$ and the total electrostatic field $\left(E_{i}\right)$ at the polarizable center, where $\mu_{i}=a_{i} \times E_{i}$, noting that the total electrostatic field:

$$
E_{i}=E_{i}^{0}+\sum_{i \neq j} T_{i j} \mu_{j} \quad \text { Equation 7.8.9 }
$$

and $T_{i j}$ is the dipole tensor:

$$
T_{i j}=\frac{1}{r_{i j}^{3}}\left(3 \vec{r}_{i j} \frac{\vec{r}_{i j}}{r_{i j}^{2}}-1\right) \text { Equation 7.8.10 }
$$

In the published literature, the inclusion of explicit polarization effects in molecular mechanics treatments has generally been applied to simulations of small polarizable molecules, ions in polarizable water, and proteins to better represent molecule association or free energies of solvation (Cieplak, 2009). More recent studies have started to address the role of polarizability in nucleic acid simulations(Babin, 2006).

A fully polarizable treatment has not been popular in large biomolecular simulations in part because adding explicit polarization tremendously increases the cost of the calculations, but also because a consistent polarizable force field for nucleic acids has not been developed. This will likely change in the near future due to the availability of faster and better methods to include explicit polarization, which in turn will lead to the development of more reliable nucleic acid force fields that are parameterized for use with explicit polarization.

\section{The Total Energy}

Added together, the two energy terms $\left(U_{\text {intra }}+U_{\text {inter }}\right)$ represent the total potential energy of the system. An important, but often overlooked, point is that it is often meaninglessdespite the use of common units for energy such as $\mathrm{kcal} / \mathrm{mol}$ - to compare absolute molecular mechanical energies between different molecules (or force fields) due to the lack of a common zero point energy. Moreover, many of the commonly employed force fields were not parameterized to very accurately estimate heats of formation, so even with a complete specification of a reaction linking two molecules together, it is likely that the relative energy between two different molecules will not be accurate. Despite this warning, it is possible to compare the relative energies of different conformations of the same molecule (under the same conditions, e.g., same force field, same number of explicit waters), remembering that this energy difference is not a free energy but only a relative energy or enthalpy. Although low energy structures are likely more representative, it is 
important to remember that this is not always the case (at normal temperatures) due to possibly large differences in entropy. For the simulation of nucleic acid systems, the most reasonable representation of the structure comes from the force fields specifically parameterized to represent nucleic acids. Current all-atom force fields for nucleic acids that perform reasonably well are described in greater detail in UNIT 7.10 and include the default nucleic acid force fields available in CHARMM and AMBER. In addition to all-atom force fields, the JUMNA internal coordinate force field and others previously mentioned also perform reasonably well.

\section{BEYOND ENERGY EVALUATION}

Evaluating the energy of a given model does not suggest anything about the relative stability or appropriateness of that model; however, differences in the relative energy between two conformations of the same model structure can suggest which structure is more enthalpically reasonable. Coupled with some representation of the relative entropy, this can give insight into the relative importance of each conformation. In general, however, it is not easy to estimate the entropy for a given conformation. Computationally demanding methods can include quasiharmonic analysis, which becomes unreliable for systems sampling multiple energy wells (Karplus and Kushick, 1981; Chang 2005). Further development of approaches to calculate configurational entropy include the nearest neighbor and mining minima methods, as well as a combination of the nearest neighbor and mutual-information expansion methods (Hnizdo et al., 2007; Chang and Gilson, 2004; Hnizdo et al., 2008); however, these are currently system size limited to short peptides. There are some more approximate methods for estimating relative conformational free energies for large systems, the best known of which (discussed in detail in UNIT 7.10) are the MM-PBSA and MMGBSA methods, ES/IS, and linear interaction energy approaches(Kollman et al., 2000, Vorobjev and Hermans, 1999, Hansson et al., 1998; Aqvist and Marelius, 2001). These methods all require knowing a priori what the representative structures are and often some parameterization or fitting.

Ideally, the investigator would like to know the conformation that represents the lowest energy (enthalpy) and ultimately the lowest free energy structures, since these structures are more representative of the true conformation of the molecule. A simple way in principle to find low-energy structures is to find the set of coordinates that minimizes the potential energy. In practice, this is limited due to the complexity of the potential energy hypersurface and the large number of degrees of freedom. In general, without exhaustive sampling of all possible conformations of a molecule it is impossible to determine if a given low-energy structure represents the true "global" minimum structure or whether it is simply a "local" minimum of the potential energy (where minimum energy structure means a structure that is at the bottom of one of many possible wells in the energy representation). Without knowing what the global minimum is, it is impossible to determine if a given model structure is at all representative of what might be expected at room temperature. Even with knowledge of the global minimum energy structure, a molecule may have a number of other low-energy structures nearby that may be populated at room temperature. 
To find representative conformations, it may be desirable to apply methods that sample according to the expected probability of observing a given conformation (at a given temperature). Examples of methods that attempt to do this are molecular dynamics (MD) or Monte Carlo (MC) methods. This is sampling according to the Boltzmann distribution and, in the limit of infinite sampling, this gives a complete representation of all the possible conformations and their relative probabilities of occurrence $\left(p_{i}\right)$, where $\varepsilon_{i}$ is the total energy of the $i^{\text {th }}$ state, $T$ is the temperature, and $k_{\mathrm{B}}$ is the Boltzmann constant.

$$
p_{i}=\frac{e^{-\varepsilon_{i} / k_{\mathrm{B}} T}}{\int e^{-\varepsilon_{i} / k_{\mathrm{B}} T}} \quad \text { Equation 7.8.11 }
$$

The term in the denominator is the partition function and specifies an integral over all phase space or the complete set of possible coordinates and momenta. Of course, in practice, sampling is limited and the sheer complexity of the accessible conformational space may prevent finding all the low-energy conformations and, therefore, full determination of the partition function. The complexity of the potential energy hypersurface and the exponential explosion of the number of possible conformations as the number of degrees of freedom increases, limit exhaustive search of this space to systems that possess only a few degrees of freedom. In 1990, none of a variety of systematic and random conformational search methods in both internal (torsion) and all-atom coordinate frames was independently able to find all the relevant low-energy conformers (within $3 \mathrm{kcal} / \mathrm{mol}$ ) of the cyclically constrained cycloheptadecane molecule, which formally has 147 degrees of freedom (all atom; Saunders et al., 1990). Today, cycloheptadecane is used as a benchmark system for testing diverse conformational search algorithms. Although computer power has increased tremendously in this time, order-of-magnitude computational speed advances do not significantly improve the effective conformational sampling of larger systems due to the exponential explosion.

A simple way to estimate the effective complexity is to assume that the number of possible minima or low-energy conformations relates to the simplistic set of three low-energy rotations about a given single bond (i.e., the trans, gauche ${ }^{+}$, and gauche $e^{-}$states) or $3^{n}-1$, where $n$ is the number of rotatable bonds. Although this is typically less than the total number of degrees of freedom, it is still large. With fewer degrees of freedom, there are less minima and sampling is easier. Moreover, minimization algorithms are less likely to become stuck in less representative local minima, though the complexity quickly grows. Even with the internal coordinate treatments for nucleic acids described above, there are still $\sim 20$ degrees of freedom per nucleotide (representing hundreds of possible conformations for a given nucleotide assuming three low-energy states for each of approximately five rotatable bonds). This places exhaustive sampling out of reach for any system with more than a few nucleotides. This difficulty in finding the global minimum (or set of coordinates that leads to the lowest energy) is often termed the multiple minima problem. In the context of the effective amount of sampling attainable during MD simulation, this problem is often termed the conformational sampling problem and relates to the improbability of overcoming large energy barriers. 
The multiple minima or conformational sampling problem is why it is desirable to choose reasonable initial structures (i.e., experimentally derived structures or valid model structures) when modeling. With reasonable structures, it is likely that MD or MC (see below) simulation will sample reasonably well near the initial structure; however, large conformational changes, such as B-DNA to Z-DNA transitions or RNA folding will not likely be seen in a reasonable time and require enhanced sampling methods. Of course, these limits in sampling imply that even with unreasonable structures, such as the imaginary and perhaps metastable B-RNA structure, MD or MC will likely sample reasonably well near the initial model structure. This is indeed the case, as shown in all-atom solvated MD simulations where B-RNA is stable for $>10 \mathrm{nsec}$ and B-RNA to A-RNA transitions are not observed unless artificial means are applied to force the conformational transition (Cheatham and Kollman, 1997). In the next sections, minimization, Monte Carlo, and molecular dynamics methods will be discussed in more detail; more detailed treatments can be found elsewhere (Valleau and Whittington, 1977; Allen and Tildesley, 1987; McCammon and Harvey, 1987; Brooks et al., 1988; van Gunsteren and Berendsen, 1990; Leach, 1997).

\section{Minimization}

Minimizing the potential energy corresponds to instantaneously "freezing" the system or dropping to the bottom of the nearest potential energy well (as shown schematically in Figure 7.8.2A). Minimization is a standard optimization problem that can be approached using tools of various complexity ranging from simple and inefficient zeroth order methods, such as grid search, which only require evaluation of the energy for a particular conformation, to more efficient but complicated $n^{\text {th }}$-order methods, which use information about all the derivatives of the energy function up to the $n^{\text {th }}$ order. Thus, to perform minimization with an $n^{\text {th }}$-order method requires analytic derivatives of the potential energy function up through the $(n-1)^{\text {th }}$ derivative (since the $n^{\text {th }}$ can be approximated by finite difference methods). In practice, a variety of first- and second-order methods are typically applied since these provide a reasonable balance between functionality (i.e. finding a minimum) and efficiency. Finding the set of coordinates that minimizes the potential energy does not guarantee that the conformation found represents the lowest energy structure due to the presence of multiple minima. As discussed, the molecule can get trapped into a local minimum which may not be representative of the "true" conformation.

In spite of the local minimum problem, minimization is still a useful tool for modeling. To eliminate potential steric overlap, or to move to geometries more consistent with the force field, or to clean up the structure after making modifications to a given model, for example the replacement of the phosphodiester backbone of DNA by a poly-amide (PNA) backbone, the conformation can be minimized. This will remove gross steric overlap and relax strained bonds and angles. While this will not say much about the relative stability of this backbone modification, it can suggest whether the backbone replacement is at least sterically feasible for the given nucleic acid conformation. Coupled with a good chemical intuition, this may provide sufficient information to the modeler to suggest whether this backbone modification is potentially useful or not. For example, consider related backbone modifications to PNA featuring either the addition or the removal of a methyl group along the backbone. Simple minimization may suggest that the shorter backbone will strain the nucleic acid, leading to a 
lower rise between base pairs, and the longer backbone will increase the separation between base pairs, which might suggest that these backbone modifications are less reasonable.

While some insight can be gained, it is important to remember that entropic effects are not included (such as the likely greater configurational entropy loss on binding for the larger PNA) and that the conformation sampled by the minimization is only a local minimum. However, if second derivatives of the potential are available, normal mode analysis can be applied to give an estimate of the entropy for the given minima in terms of rotational, translation and vibrational degrees of freedom (Allen and Tildesley, 1987). In addition to being a useful tool for modeling, minimization is necessary prior to running molecular dynamics simulations to fix up the positioning of added hydrogens consistent with SHAKE constraints, to relieve any large steric overlaps, or to remove strained bonds or angles that might otherwise lead to initially large forces. During molecular dynamics, large initial forces can lead to significant atomic displacements, which in turn contribute to more steric overlaps and increasingly large displacements; this cascade of events can lead to local hot spots or failure of the integrator during MD.

There are two common first-order minimization methods in common usage: the steepest descent (Wiberg, 1965) and conjugate gradient (Fletcher and Reeves, 1964) methods. These use the first derivative to give information about the slope of the potential (but not the curvature). In steepest descent, movement is made parallel to the net force. Given reasonable step sizes, this method will not cross barriers and will readily traverse down to the bottom of the nearest potential energy well. Although this method is not very efficient, it is very stable and is therefore often used to initially minimize the structure when there are large energies. Often this is used as the first step in modeling, particularly when there are potentially large van der Waals overlaps to initially relax the most drastic energetic penalties. The conjugate gradient method improves upon the steepest descent by using the gradients from previous steps to further guide the minimization. This method is more efficient than steepest descent and is appropriate to apply after initial minimization (e.g., to remove the largest steric overlaps). Near the bottom of the harmonic well, or after some initial minimization, use of second order methods (which assume an approximately quadratic relationship to the energy) such as Newton-Raphson (TNPACK; Schlick and Overton, 1987) can be used to speed up convergence at the expense of greater memory usage and the need for second derivatives (either by finite difference or analytical). These methods are typically more expensive since they require inverting the second derivative matrix. The expense can be significantly reduced by limiting the space sampled to regions where there is significant movement in the energy, thus limiting the size of the second derivative matrix (which is calculated by finite difference; Brooks et al., 1983) and memory footprints can be optimized (Liu and Nocedal, 1989). Recent and efficient minimizers include the XMIN minimizer available within the freely available Amber Tools suite of programs.

The key point is that minimization is a very useful tool, but that care should be taken when analyzing the validity of a particular "low-energy" structure. Because of the limits in sampling and the great likelihood of minimizing to a local minimum (which may or may not be the representative low-energy structure of the ensemble), the validity needs to be judged based on the chemical intuition of the modeler and known reliability of the initial model. To 
better sample space, Monte Carlo or molecular dynamics methods can be applied; although these methods avoid the problem of getting trapped in local minima, limits in sampling only allow partial sampling of conformations "near" the initial structure. Despite the limited sampling, minimization is an extremely useful tool to characterize model structures.

Note that care should be taken when applying restraints along with minimization, such as when using NMR-derived distance restraints, to balance the restraint force constants with those of the force field. If the restraint force constants are too large relative to the force field, unrealistic distortion of the structure may result, including flips in chirality. As a final practical comment about minimization, it is important to initially use small step sizes during the minimization of high-energy structures. This prevents the minimizer from jumping out of the current well to a potentially higher energy surface, which with repeated large jumps can lead to instability. Moreover, if the step size is too large, the minimizer may effectively get "trapped" in a cycle, jumping back and forth across the bottom of the well there by preventing the minimizer from converging.

\section{Monte Carlo}

The Metropolis Monte Carlo method is essentially an algorithm for generating a random walk in conformational space such that the conformations obtained are distributed according to the probabilities expected for the equilibrium Boltzmann distribution (Metropolis et al., 1953; Valleau and Whittington, 1977). The algorithm is very simple and requires a single evaluation of the potential energy (and no force calculation) for each step (Figure 7.8.2B). At each step, a random movement is made. In an all-atom representation, this may be the movement of a single particle; with an internal coordinate representation, it is a movement along the normal coordinates (such as a rotation of one of the rigid units). The potential energy difference $(\Delta E)$ between the new conformation and the old is calculated. If the energy of the new conformation is lower, the move is accepted. If not, the move is accepted if a random number drawn from the interval $[0,1]$ is less than $\mathrm{e}^{-\Delta \mathrm{E} / \mathrm{k}_{\mathrm{B}} T}$; otherwise the old conformation is retained. The length of the simulation relates to the number of attempted moves or configurations. The length of the simulation does not relate to the time scale and only represents the number of configurations sampled.

Use of this procedure leads to a representative set of structures. With sufficient sampling, this will converge to the equilibrium distribution and in principle allow reasonable estimation of any ensemble average, assuming the forces between particles are velocity independent (Metropolis et al., 1953). This assumption allows one to separate out the momenta or kinetic energy from the total energy such that only the potential energy is evaluated in the above expression; in practice, the kinetic component is always ignored. Since uphill moves are only accepted randomly (and not according to any deterministic process), there is no implicit time evolution and the progression of the sampling gives no information about the dynamics. The success of this procedure (with finite sampling) largely relates to the "move set", or set of possible moves. When an all-atom treatment is applied with single particle moves, the stiff internal degrees of freedom can lead to large energy changes and therefore a small step size needs to be used. With small step sizes, many more configurations may need to be evaluated to generate an appropriate ensemble, decreasing the 
efficiency. Moreover, for systems with disparate frequencies in different degrees of freedom -i.e., both stiff internal degrees of freedom (bonds, angles) and softer modes (correlated movements, dihedral rotation) — such as nucleic acids or single particle or atom moves, this leads to poor sampling. Even for liquid simulation, small atom-based moves lead to low acceptance ratios and poor sampling. In liquid simulation this can be overcome by using moves along normal coordinates, such as bond rotations, coupled with rotations and translations of the entire molecule. In general, it is desirable to avoid move sets that overly reject moves, and a roughly $40 \%$ acceptance rate represents a reasonable balance (Jorgensen and Tirado-Rives, 1996).

The difficulty in choosing a proper move set led to the early impression that MD simulations were up to ten times more efficient than $\mathrm{MC}$ at generating conformations of the small protein BPTI (Northrup and McCammon, 1980); however, this simulation used an extremely inefficient move set based on movement of atomic centers. Much better behavior is seen with a move set based on internal coordinate rotations about bonds (with rigid bond length and angles; Noguti and Go, 1985). For liquid simulation, $\mathrm{MC}$ is likely the most efficient method for generating reasonable ensembles; an excellent MC program for liquid simulation is BOSS developed by the Jorgensen group (Jorgensen, 1995). Use of the MC procedure for the simulation of neat liquids led to the development of very reliable van der Waals parameters for the OPLS force field (Jorgensen et al., 1996). A direct comparison of MC and MD simulation of liquid hexane suggests that $\mathrm{MC}$ is roughly three times more efficient than MD when an appropriate move set for the MC is applied (Jorgensen and Tirado-Rives, 1996).

Although MC is more efficient for liquid simulations, it is not clear this generalization follows to the sampling the potential energy landscape of large biomolecules, particularly in solution. For example, with internal coordinate moves with flexible long-chain molecules (such as nucleic acids), a small rotation about a central dihedral angle can lead to a large displacement of the end of the chain. In explicit water, this could lead to extreme van der Waals overlap and likely very high rejection rates (for these types of moves). Although the use of correlated moves (such as crankshaft rotations about two bonds) can counter this effect (Dodd et al., 1993; Deem and Bader, 1996), there has been little published use of MC methods in all-atom simulation of nucleic acids in explicit solvent. For simulations without explicit solvent, MC simulation is widely used for nucleic acid simulation particularly with internal coordinate representations. Examples include its use in structure predictions as previously mentioned (Erie et al., 1993), investigating DNA bending in polyadenine tracts (Zhurkin et al., 1991), investigating counterion distribution about DNA (Young et al., 1997), and investigating the kinetics of hairpin folding (Sauerwine, 2011).

\section{Molecular Dynamics}

Molecular dynamics simulation refers to integrating numerically the classical equations of motion (Newton's equations) for all the atoms in the system (Figure 7.8.2C). A simulation is started by assigning random momenta (velocities, $v_{i}$ ) for each of the $N$ particles (of mass $m_{i}$ ) from a Maxwell-Boltzmann distribution about a given temperature, $T$, where the temperature is defined as below. 


$$
\frac{1}{2} \sum_{i}^{N} m_{i} v_{i}^{2}=\frac{3 N k_{\mathrm{B}} T}{2} \quad \text { Equation 7.8.12 }
$$

Then the dynamics are propagated by integrating Newton's equations of motion, which for the pairwise potential, $U_{i}$, and its first derivative:

$$
\nabla_{i} \sum_{j}^{N} U_{i j} \quad \text { Equation 7.8.13 }
$$

is represented by the following:

$$
m_{i} \frac{d^{2} r_{i}}{d t^{2}}=-\nabla_{i} \sum_{j}^{N} U_{i j} \quad \text { Equation 7.8.14 }
$$

The integration is typically performed through the use of one of a variety of first-order integration algorithms, such as leap-frog or velocity Verlet which are variations on the original Verlet scheme (Allen and Tildesley, 1987). This requires the calculation of the forces at each step, so a typical MD step involves calculation of the energy, forces, and velocities, and integration to obtain the coordinates for the next step. An important assumption is the ergodic hypothesis that, in the limit of complete sampling, the time average (that obtained by molecular dynamics) is equivalent to the ensemble average. Given sufficient sampling, it appears that the ergodic hypothesis is valid in practice since both MD and $\mathrm{MC}$ lead to equivalent ensembles for liquid hexane even though they use rather different sampling mechanisms (Jorgensen and Tirado-Rives,1996). Also note that, unlike MC, the MD sampling is based on a dynamic propagation of the molecular mechanical forces; therefore, it is important that the forces are analytical or exact derivatives of the energy (i.e., the forces and energies should match). This is not always true in practice since the force calculation in some cases may be too expensive (particularly for non-pair wise forces), requiring some approximations. This means that the sampling is done on a surface different than the molecular mechanical energy surface.

Issues in MD simulation include the need for stable, reversible, and ideally symplectic (which implies loosely that the algorithm conserves energy and momentum) integrators. The simple first-order Verlet, velocity Verlet, and leap-frog integration algorithms satisfy these conditions in proper usage, in contrast to the more complex higher-order integrators such as the Gear predictor. Stability of the integration directly relates to the integration time step that in turn relates to the expected frequency of motion. A simple rule of thumb for Verlet integrators is that the integration catastrophe, or the time step where the integrator blows up, is the period of the highest frequency motion divided by $\pi$. For an all-atom simulation, the highest-frequency motions involve bond stretching of bonds to hydrogen. In the absence of rigid bond lengths (or constrained bonds), time steps are limited to the $\sim 1$ fsec range. With SHAKE (Ryckaert et al., 1977) applied to constrain the lengths of bonds to hydrogen, time steps in the 2 fsec range are routinely applied. Larger time steps are possible by limiting the high-frequency motion, such as through the use of rigid units, which then necessitates 
inverting the inertia tensor as with internal coordinate treatments or the need for imposition of iteratively solved holonomic constraints. Effectively larger time steps are also possible through the application of multiple time step methods, which treat the slowly varying forces (which ideally represent the more computationally demanding part of the energy and force evaluation) with a longer time step, such as the long-range pairwise forces (Tuckerman et al., 1992; Biesiadecki and Skeel, 1993). With increased masses on hydrogen atoms to limit high-frequency motion, time steps as large as $5 \mathrm{fsec}$ have been applied to systems with explicit water, and many current MD codes have implemented hydrogen atom mass repartitioning algorithms to speed up the calculations. Coarse grain simulations may allow time steps of up to 10fs (Winger, 2009).

The symplectic nature of the integrator relates to preserving the Hamiltonian (or loosely the energy representation) of the system during integration. Not only should this be a property of the integrator, but molecular dynamics simulation in general should conserve energy. This is an excellent test of the methods. Note that in common usage, MD programs do not always necessarily conserve energy. This is often due to SHAKE tolerances that are not stringent enough, integration time steps that are too large, the use of the weak-coupling algorithm for constant pressure, and neglect of pair interactions. In order to speed the calculation, the effective number of pair interactions is often reduced to only those within a given range and a list of in-range pair interactions is maintained for each atom. For speed this "pair list" is not updated every step. Unless a buffer is maintained to not omit (include) pair interactions moving into (out of) range which is conservatively or heuristically updated, energy conservation may not be maintained. How the pair interactions are limited to finite range can also have important consequences, as can temperature or pressure coupling for constant T, P ensembles. In order to deterministically integrate Newton's equations of motion, it is important that systematic force errors be avoided (such as can occur with lack of energy conservation and temperature scaling) since these can lead to behavioral artifacts, such as violation of equipartition (Harvey et al., 1998; Chiu et al.,2000). For example, with energy drains and the application of temperature adjustment by uniform scaling of the velocities of all the atoms, energy accumulates in low-frequency modes. This can lead to a growth of the center of mass translation. Given this, and since random velocity assignment likely leads to nonzero center of mass translational motion, it is advisable to remove this motion at the beginning of an MD run. Random force errors, such as those resulting from the slow accumulation of errors due to finite numerical precision, do not seem to lead to artifacts since they are equally likely to add as subtract from the total energy. To this end, differences obtained between sequential and parallel MD runs due to differences in the order of operations do not lead to significant differences and simply manifest the inherently chaotic nature of the integration (Braxenthaler et al., 1997). Yet, as many groups try to produce the fastest MD codes on parallel, GPU, and special purpose resources, often approximations are made which may impact accuracy, energy conservation, and effective sampling in MD simulation.

Variants of standard MD include Langevin and Brownian dynamics. These MD methods are used to implicitly include the diffusive effects of solvent. In practice, this involves adding to the standard forces an additional set of stochastic forces from a heat bath (via random fluctuation of forces) along with dissipative forces to balance them. For in vacuo 
simulations, this represents thermal collisions with other molecules and allows coupling of energy among the different internal degrees of freedom. This can be very useful since during deterministic dynamics without Langevin, memory of the initial conditions may persist in the form of various correlated motions, and some low-frequency correlated motions may not be able to couple back into other modes of motion (since there are no collisions with other molecules) particularly in solutions without inclusion of explicit solvent. This can lead to poor sampling. A caveat in the implementation of Langevin dynamics algorithms concerns initial velocity assignments, which are performed using random number generators. It has been shown that rapid restarts using the same seed for a random number generator results in residual stochastic force (Cerutti et al., 2008). Additionally, use of the same seed for different trajectories leads to a synchronization bias across trajectories, even at different temperatures and for large systems, and this can lead to, for example, spontaneous melting of DNA duplexes (Sindhikara et al., 2009, Uberaga et al., 2004). In the limit of very high friction, Langevin dynamics become Brownian dynamics. Brownian dynamics are purely diffusive and effectively add a random coordinate displacement. This method is typically used to model the diffusional motion of molecules (such as the encounter of a substrate into an enzyme binding site) and with coarse-grained force field models. More detailed discussion of MD and its variants can be found in the previously cited books and a very useful published discussion of stochastic dynamics methods (Pastor, 1994).

With molecular dynamics, each particle has a finite kinetic energy; a direct implication is that the rate of barrier crossing will be proportional to the temperature and the length of the simulation. This implies that the probability of crossing large energy barriers during MD is rather small (as is represented schematically in Figure 7.8.2C). A back-of-the-envelope estimation of the rate of barrier crossing can be obtained from transition state theory with some basic approximations.

$$
k \cong \kappa v \times e^{-G_{a c t}^{\grave{\ddagger}} / R T} \quad \text { Equation 7.8.15 }
$$

The rate $k$ is related to the transmission coefficient $\kappa$, which represents the ratio of successful transitions over the barrier, an effective rate at the top the barrier or equivalently a factor loosely representing collisions with the barrier, $\nu$, times a Boltzmann-weighted "free energy of activation," which represents the height of the barrier. For simplicity, it can be assumed that at the top of the barrier, the particle always crosses the barrier rather than reflecting back, leading to $K=1$ (in contrast to expected values of 0.4 to 1.0 in solution). By classical equipartition $\left(E \cong k_{\mathrm{B}} T\right)$ and from $E=h \nu$ this barrier rate is approximately:

$$
\nu=\frac{k_{\mathrm{B}} T}{h} \quad \text { Equation 7.8.16 }
$$

where $T$ is the temperature, $h$ is Plank's constant, and $k_{\mathrm{B}}$ is the Boltzmann constant. At room temperature, $R T$ is $\sim 0.6 \mathrm{kcal} / \mathrm{mol}$ and $v$ is $\sim 6.2 \mathrm{psec}^{-1}$. Remembering that this is only an approximation, this suggests that barriers of $\sim 1 \mathrm{kcal} / \mathrm{mol}$ can be surmounted in picoseconds $\left(\sim 1.2 \mathrm{psec}^{-1}\right)$ and $\sim 5 \mathrm{kcal} / \mathrm{mol}$ in nanoseconds $\left(\sim 1.5 \mathrm{nsec}^{-1}\right)$, but that barriers $>10 \mathrm{kcal} / \mathrm{mol}$ may take microseconds or longer. This relates to the conformational sampling problem and is a significant limitation of MD. In MC simulations, this problem can be overcome by 
increasing the size of the moves (at the expense of lower acceptance ratios) or by designing clever move sets. In a similar manner, with MD simulation various methods can be applied to effectively lower barriers to conformational transition, such as adding biasing potentials to lower specific torsion barriers, increasing masses, increasing temperature, smoothing the overall potential surface, or applying mean field approximations, such as with the locally enhanced sampling (LES) methodology. The LES methodology reduces the barriers to conformational transition, thereby allowing transition from an "incorrect" to correct RNA hairpin loop conformation (Simmerling et al., 1998). Also very popular in recent years is the application of replica-exchange or parallel tempering methods where multiple independent simulations are run simultaneously (Swendsen and Wang, 1986, Sugita and Okamoto, 1999), each swapping particular process variables, such as temperature or Hamiltonian (Curuksu and Zacharias, 2009), according to a well-defined Metropolis criteria. Such methods can sample conformational ensembles more effectively than standard MD simulation, although complete convergence of RNA tetraloop geometries is still a challenge (Garcia and Paschek, 2008, Zuo et al., 2010, Kuhrova et al, 2013) and convergence of the complete conformational distribution of an RNA tetranucleotide, $\mathrm{r}(\mathrm{GACC})$, still requires $\sim 2$ $\mu$ s per replica (24 or 36 replicas) in standard temperature replica-exchange (Henriksen et al, 2013). A further issue, for duplex or multimeric nucleic acid systems is that temperature based replica-exchange will tend to irreversibly unfold the multimer on standard simulation time scales suggesting that Hamiltonian replica-exchange methods may be more promising. For reviews of enhanced sampling methods, see Schlick (2009) and Straub (1996).

The attainable MD simulation time scale relates to the complexity of the potential (and therefore the time required to evaluate the energy and forces) and the integration time step. With longer time steps, longer simulations are possible. Through the deterministic procedure and specification of an integration time step, there is a direct relationship between the number of MD steps and the effective time scale (time step $\times$ the number of steps $=$ total time). The current state of the art for MD simulation of nucleic acids in explicit solvent involves simulations over 1 microsecond on 12-mer to 18-mer duplexes (Perez, 2007, Beveridge et al., 2012), with scales of 1-40 $\mu$ s easily obtainable today on GPU or specialized resources such as D.E. Shaw's Anton machine. Although in principle the time scale is "accurately" represented by the dynamics or integration of the classical equations of motion, whether a $1 \mathrm{nsec}$ simulation of DNA actually accurately represents $1 \mathrm{nsec}$ of real motion depends on the empirical potential employed. For equilibrium or ensemble properties (such as the energy, free energy, heat capacity, or density) this exact time scale is not important, assuming independence of the forces on the velocity of a given particle. This is implicitly the case with the standard molecular mechanics potentials. For ensemble properties, what matters is the effective amount of sampling. In principle, one can obtain equivalent ensemble averages even if the masses on all the hydrogens are increased. This will allow a larger time step and therefore longer sampling. Although this does not affect the ensemble properties, this will drastically affect time-averaged properties, such as water diffusion or the rate of specific conformational transitions.

The point is that these dynamic properties are very sensitive to the potential (and atomic masses). Since the force field is primarily parameterized to represent structure, the dynamic properties are subjective and more dependent on the choice of force field and solvent 
models. Clearly in the absence of viscous damping forces or explicit solvent, the rate of dynamics may be enhanced relative to simulations in explicit solvent. Similarly, under high pressure conditions, such as in an isolated solvent droplet, the dynamics may be reduced. In explicit solvent, MD simulations of proteins and nucleic acids display thermal parameters that are in good accord with experiment and in general properly represent fast (picosecond) time scale motions. Comparison to experiment is critical, and this becomes more tractable as longer time scales are attained. Evidence from the simulations is that some properties are well represented; however, there is a clear overestimation of harmonic motion in solvated proteins at low temperature (i.e., suggesting motion that is too slow(Steinbach and Brooks, 1994). On the other hand, one of the most commonly used water models, TIP3P (Jorgensen et al., 1983) diffuses at twice the experimental value. The point of this discussion of time scale and dynamics is not to criticize the methods but to remind potential modelers of the various issues and sensitivity not only to the force field but the representation. Although in general the results are within range, it is important to understand how the methods have been validated by comparison to experiment for a given property before making firm claims about exact details of the time scale for conformational transition. For example, estimating the free energy of binding for a particular water molecule to DNA based on the lifetime of its bound state is likely to be inaccurate and very dependent on the specific water model, and the type of water model used has been shown to affect simulations of RNA (Sklenovsky 2011).

Additionally, in order to make claims about the time scale for a given process based on MD, the time scale of the simulation should be significantly longer than the relaxation time of the process of interest. Likely the simulation should be at least an order, if not two orders, of magnitude longer than the relaxation time and, when attempting to make statistically valid claims about the rate of a given transition or specific correlation, many events need to be observed. These and related issues regarding the validation of simulation results are presented in a classic review (van Gunsteren and Mark, 1998).

\section{SUMMARY}

In this unit, the basic principles of the common energy representations for nucleic acid models have been presented, and methods for exploring these energy surfaces have been discussed in some detail. To move beyond simple energy evaluation of a single model structure, it is fairly clear that a large number of energy (and possibly force) evaluations will be necessary. This becomes computationally demanding, and it is desirable to limit the computational cost as much as possible without sacrificing accuracy. For systems of reasonable size, there is a need to use empirical potentials as discussed; however, even with the simple pairwise potentials, the number of pair interactions quickly grows as the number of atoms is increased. Thus, for larger systems the effective number of intermolecular interactions is reduced by limiting the range of the interaction (e.g., by limiting interactions to distances less than some cutoff or utilizing hierarchical approaches that coalesce groups of distant atoms into an approximate single effective particle). Additional complications relate to the representation of nucleic acids since water and associated counterions (salt) are an integral part of nucleic acid structure (see UNIT 7.9). 


\section{Acknowledgments}

This is an update to the original protocols article by T.E. Cheatham, III, B.R. Brooks and P.A. Kollman (Current Protocols in Nucleic Acid Chemistry, UNIT 7.8, 2001). We would like to acknowledge Sean Cornillie, Hamed Hayatshahi, Niel Henriksen, and Dan Roe for critical readings of the manuscript and also to acknowledge funding from the NIH to TEC3 through R-01 GM081411 and GM098102.

\section{LITERATURE CITED}

Abolfath RM, Biswas PK, Rajnarayanam R, Brabec T, Kodym R, Papiez L. Multiscale QM/MM molecular dynamics study on the first steps of guanine damage by free hydroxyl radicals in solution. J Phys Chem A. 2012; 15:3940-5. [PubMed: 22397677]

Allen, MP.; Tildesley, DJ. Computer Simulation of Liquids. Oxford University Press; Oxford: 1987.

Allinger NL. Conformational analysis 130.MM2. A hydrocarbon force field utilizing V1 and V2 torsional terms. J Am Chem Soc. 1977; 99:8127-8134.

Allinger NL, Yuh YH, Lii JH. Molecular mechanics. The MM3 force field for hydrocarbons.1. J Am Chem Soc. 1989; 111:8551-8566.

Allinger NL, Chen K, Lii JH. An improved force field (MM4) for saturated hydrocarbons. J Comp Chem. 1996; 17:642-668.

Aqvist J, Marelius J. The linear interaction energy method for predicting ligand binding free energies. Comb Chem High T Scr. 2001; 14:613-626.

Babin V, Baucom J, Darden TA, Sagui C. Molecular dynamics simulations of DNA with Polarizable force fields: convergence of an ideal B-DNA structure to the crystallographic structure. J Phys Chem B. 2006; 110:11571-11581. [PubMed: 16771434]

Baker CM, Anisimov VM, MacKerell AD Jr. Development of CHARMM polarizable force field for nucleic acid bases based on the classical Drude oscillator model. J Phys Chem B. 2011; 115:580596. [PubMed: 21166469]

Banas P, Jurecka P, Walter NG, Sponer J, Otyepka M. Theoretical studies of RNA catalysis: hybrid QM/MM methods and their comparison with MD and QM. Methods. 2009; 49:202-216. [PubMed: 19398008]

Beveridge DL, Cheatham TE III, Mezei M. The ABCs of molecular dynamics simulation on B-DNA, circa 2012. J Biosci. 2012; 37:379-397. [PubMed: 22750978]

Biancardi A, Biver T, Secco F, Mennucci B. An investigation of the photophysical properties of minor groove bound and intercalated DAPI through quantum-mechanical and spectroscopic tools. Phys Chem Chem Phys. 2013; 15:4596-4603. [PubMed: 23423468]

Biesiadecki JJ, Skeel RD. Dangers of multiple time step methods. J Comp Phys. 1993; 109:318-328.

Braxenthaler M, Unger R, Auerbach J, Given A, Moult J. Chaos in protein dynamics. Proteins. 1997; 29:417-425. [PubMed: 9408939]

Brooks BR, Brooks CL, Brooks III, Mackerell AD, Nilsson L, Petrella RJ, Roux B, Won Y, Archontis G, Bartels C, Boresch S, Caflisch A, Caves L, Cui Q, Dinner AR, Feig M, Fischer S, Gao J, Hodoscek M, Im W, Kuczera K, Lazaridis T, Ma J, Ovchinnikov V, Paci E, Pastor RW, Post CB, Pu JZ, Schaefer M, Tidor B, Venable RM, Woodcock HL, Wu X, Yang W, York DM, Karplus M. CHARMM: The Biomolecular simulations Program. J Comp Chem. 2009; 30:1545-1615. [PubMed: 19444816]

Brooks, CL., III; Karplus, M.; Pettitt, BM. Proteins. A Theoretical Perspective of Dynamics, Structure, and Thermodynamics. John Wiley \& Sons; New York: 1988.

Bruccoleri RE, Olafson BD, States D, Swaminathan JS, Karplus M. CHARMM: A program for macromolecular energy, minimization, and dynamics calculations. J Comp Chem. 1983; 4:187217.

Caldwell JW, Dang LX, Kollman PA. Implementation of nonadditive intermolecular potentials by use of molecular dynamics: Development of a water-water potential and water-ioncluster interactions. J Am Chem Soc. 1990; 112:9144-9147. 
Cances E, Mennucci B, Tomasi J. A new integral equation formalism for the polarizable continuum model: theoretical background and applications to isotropic and anisotropic dielectrics. J Chem Phys. 1997; 107:3032-3041.

Case DA, Cheatham TE III, Darden T, Gohlke H, Luo R, Merz KM Jr, Onufriev A, Simmerling C, Wang B, Woods R. The Amber biomolecular simulation programs. J Computat Chem. 2005; 26:1668-1688.

Cerutti DS, Duke R, Freddolino PL, Fan H, Lybrand TP. A vulnerability in popular molecular dynamics packages concerning Langevin and Andersen dynamics. J Chem Theory Comput. 2008; 4:1669-1680. [PubMed: 19180249]

Chatfield DC, Eurenius KP, Brooks BR. HIV-1 protease cleavage mechanism: Atheoretical investigation based on classical MD simulation and reaction path calculations using a hybrid QM/MM potential. Theochem J Mol Struct. 1998; 423:79-92.

Chang CE, Chen W, Gilson MK. Evaluating the accuracy of the quasiharmonic approximation. J Chem Theory Comput. 2005; 1:1017-1028.

Chang CE, Gilson MK. Free energy, entropy, and induced fit in host-guest recognition: calculations with the second-generation mining minima algorithm. J Am Chem Soc. 2004; 40:13156-13164. [PubMed: 15469315]

Cheatham TE III, Kollman PA. Molecular dynamics simulations highlight the structural differences in DNA:DNA, RNA:RNA and DNA:RNA hybrid duplexes. J Am Chem Soc. 1997; 119:4805-4825.

Cheatham TE III, Cieplak P, Kollman PA. A modified version of the Cornell et al. force field with improved sugar pucker phases and helical repeat. J Biomol Struct Dyn. 1999; 16:845-862. [PubMed: 10217454]

Chiu SW, Clark M, Subramaniam S, Jakobsson E. Collective motion artifacts arising in long-duration molecular dynamics simulations. J Comp Chem. 2000; 21:121-131.

Cieplak P, Dupradeau FY, Duan Y, Wang J. Polarization effects in molecular mechanics force fields. J Phys Condens Matter. 2009; 21:333102-333123. [PubMed: 21828594]

Cornell WD, Cieplak P, Bayly CI, Gould IR, Merz KM, Ferguson DM, Spellmeyer DC, Fox T, Caldwell JW, Kollman PA. A second generation force field for the simulation of proteins, nucleic acids, and organic molecules. J Am Chem Soc. 1995; 117:5179-5197.

Cummins PL, Gready JE. Coupled semiempirical molecular orbital and molecular mechanics model (QM/MM) for organic molecules in aqueous solution. J Comp Chem. 1997; 18:1496-1512.

Curuksu J, Zacharias M. Enhanced conformational sampling of nucleic acids by a new Hamiltonian replica exchange molecular dynamics approach. J Chem Phys. 2009; 130:104110. [PubMed: 19292526]

Deem MW, Bader JS. A configurational bias Monte-Carlo method for linear and cyclic peptides. Mol Phys. 1996; 87:1245-1260.

de Pablo JJ. Coarse-grained simulations of macromolecules: from DNA to nanocomposites. Ann Rev Phys Chem. 2011; 62:555-574. [PubMed: 21219152]

Denning EJ, Priyakumar UD, Nilsson L, Mackerell AD Jr. Impact of $2^{\prime}$-hydroxyl sampling on the conformational properties of RNA: update of the CHARMM all-atom additive force field for RNA. J Comput Chem. 2011; 32:1929-1943. [PubMed: 21469161]

Dixon SL, Merz KMJ. Fast, accurate semiempirical molecular orbital calculations for macromolecules. J Chem Phys. 1997; 107:879-893.

Dodd LR, Boone TD, Theodorou DN. A concerted rotation algorithm for atomistic Monte-Carlo simulation of polymer melts andglasses. Mol Phys. 1993; 78:961-996.

Erie DA, Breslauer KJ, Olson WK. A Monte Carlo method for generating structures of short singlestranded DNA sequences. Biopolymers. 1993; 33:75-105. [PubMed: 8427940]

Field M, Bash P, Karplus M. A combined quantum mechanical and molecular mechanical potential for molecular dynamics simulation. J Comp Chem. 1990; 11:700-733.

Flatters D, Zakrzewska K, Lavery R. Internal Coordinate Modeling of DNA: Force Field Comparisons. J Comp Chem. 1997; 18:1043-1055.

Fletcher R, Reeves CM. Function minimization by conjugate gradients. Computer J. 1964; 7:149-153. 
Flores SC, Sherman MA, Bruns CM, Eastman P, Altman RB. Fast flexible modeling of RNA structure using internal coordinates. IEEE/ACM Trans Comput Biol Bioinfom. 2011; 8:1247-1257.

Foloppe N, MacKerell ADJ. All-atom empirical force field for nucleic acids. 1) Parameter optimization based on small molecule and condensed phase macromolecular target data. J Comp Chem. 2000; 21:86-104.

Garcia AE, Paschek D. Simulation of the pressure and temperature folding/unfolding equilibrium of a small RNA hairpin. J Amer Chem Soc. 2008; 130:815-817. [PubMed: 18154332]

Gao JL. Hybrid quantum and molecular mechanical simulations-An alternative avenue to solvent effects in organic chemistry. Acc Chem Res. 1996; 29:298-305.

Gorin AA, Ulyanov NB, Zhurkin VB. S-N transition of the sugar ring in B-form DNA. Molekulyarnaya Biologiya. 1990; 24:1300-1313.

Grimme S. Density functional theory with London dispersion corrections. Wiley Interdisciplinary Reviews: Computational Molecular Science. 2011; 1:211-228.

Halgren TA. Merck molecular force field 1. Basis, form, scope, parameterization, and performance of MMFF94. J Comp Chem. 1996; 17:490-519.

Hansson T, Marelius J, Aqvist J. Ligand binding affinity prediction by linear interaction energy methods. J Comput Aid Mol Des. 1998; 12:27-35.

Hart K, Foloppe N, Baker CN, Denning EJ, Nilsson L, Mackerell AD Jr. Optimization of the CHARMM additive force field for DNA: improved treatment of the BI/BII conformational equilibrium. J Chem Theory Comput. 2012; 8:348-362. [PubMed: 22368531]

Harvey SC, Tan RKZ, Cheatham TE III. The flying ice cube: Velocity rescaling inmolecular dynamics simulations leads to violation of equipartition. J Comp Chem. 1998; 19:726-740.

Harvey SC, Wang C, Teletchea S, Lavery R. Motifs in nucleic acids: Molecular mechanics restraints for base pairing and base stacking. J Comp Chem. 2003; 24:1-9. [PubMed: 12483670]

Henriksen NM, Roe DR, Cheatham TE III. Reliable oligonucleotide conformational ensemble generation in explicit solvent for force field assessment using reservoir replica exchange molecular dynamics simulations. J Phys Chem B. 2013; 117:4014-4027. [PubMed: 23477537]

Hess B, Kutzner C, van der Spoel D, Lindahl E. GROMACS 4: Algorithms for Highly Efficient, LoadBalanced, and Scalable Molecular Simulation. J Chem Theory Comput. 2008; 3:435-447.

Hobza P, Sponer J. Structure, energetics, and dynamics of the nucleic acid base pairs: Nonempirical ab initio calculations. Chem Rev. 1999; 11:3247-3276. [PubMed: 11749516]

Hobza P, Kabelac M, Sponer J, Mejzlik P, Vondrasek J. Performance of empirical potentials (AMBER, CFF95, CVFF, CHARMM, OPLS, POLTEV), semiempirical quantum chemical methods (AM1, MNDO/M, PM3), and ab initio Hartree-Fock method for interaction of DNA bases: Comparison with nonempirical beyond Hartree-Fock results. J Comp Chem. 1998; 18:1136-1150.

Hnizdo V, Darian E, Fedorowicz A, Demchuk E, Li S, Singh H. Nearest-neighbor nonparametric method for estimating the configurational entropy of complex molecules. J Comp Chem. 2007; 28:655-668. [PubMed: 17195154]

Hnizdo V, Tan J, Killian BJ, Gilson MK. Efficient calculation of configurational entropy from molecular simulations by combining the mutual-information expansion and nearest-neighbor methods. J Comp Chem. 2008; 29:1605-1614. [PubMed: 18293293]

Jorgensen, W. BOSS, Version 3.6. Yale University; New Haven: 1995.

Jorgensen WL, Tirado-Rives J. Monte Carlo vs Molecular dynamics for conformational sampling. J Phys Chem. 1996; 100:14508-14513.

Jorgensen WL, Chandrasekhar J, Madura JD, Impey RW, Klein ML. Comparison of simple potential functions for simulating liquid water. J Chem Phys. 1983; 79:926-935.

Jorgensen WL, Maxwell DS, Tirado-Rives J. Development and testing of the OPLS all-atom force field on conformational energetics and properties of organic liquids. J Am Chem Soc. 1996; 118:11225-11236.

Karplus M, Kushick JN. Method for estimating the configurational entropy of macromolecules. Macromolecules. 1981; 14:325-332. 
Klamt A, Schuurmann G. COSMO: A new approach to dielectric screening in solvents with explicit expressions for the screening energy and its gradient. J Chem Soc Perkin Trans 2. 1993; 5:799_ 805 .

Kollman PA, Massova I, Reyes C, Kuhn B, Huo S, Chong L, Lee M, Lee T, Duan Y, Wang W, Donni D, Cieplak P, Srinivasan J, Case DA, Cheatham TE III. Calculating structures and free energies of complex molecules: Combining molecular mechanics and continuum models. Acc Chem Res. 2000; 33:889-897. [PubMed: 11123888]

Korth M. Third-generation hydrogen-bonding corrections for semiempirical QM methods and force fields. J Chem Theory Comput. 2010; 6:3808-3816.

Korth M, Pitonak M, Rezac J, Hobza P. A transferable h-bonding correction for semiempirical quantum-chemical methods. J Chem Theory Comput. 2010; 6:344-352.

Kuhrova J, Banas P, Best RE, Sponer J, Otyepka P. Computer folding of RNA tetraloops? Are we there yet? J Chem Theory Comp. 2013; 9:2115-2125.

Lahiri A, Nilsson L. Properties of dianionic oxyphosphorane intermediates from hybrid QM/MM simulations: Implications for ribozyme reactions. J Mol Struct. 1997; 419:51-55.

Langley DR. Molecular dynamics simulations of environment and sequence dependent DNA conformation: The development of the BMS nucleic acid force field and comparison with experimental results. J Biomol Struct Dyn. 1998; 16:487-509. [PubMed: 10052609]

Lavery R, Zakrzewska K, Sklenar H. JUMNA (junction minimisation of nucleic acids). Comp Phys Comm. 1995; 91:135-158.

Leach, AR. Molecular Modeling: Principles and Applications. Addison-Wesley; Reading, Mass: 1997.

Lee TS, Silva Lopez C, Giambasu GM, Martick M, Scott WG, York DM. Role of Mg2+ in hammerhead ribozyme catalysis from molecular simulation. J Amer Chem Soc. 2008; 130:30533064. [PubMed: 18271579]

Levine, IN. Quantum Chemistry. Prentice Hall; Englewood Cliffs, N.J: 1991.

Lior-Hoffmann L, Wang L, Wang S, Geacintov NE, Broyde S, Zhang Y. Preferred WMSA catalytic mechanism of the nucleotidyl transfer reaction in himan DNA polymerase K elucidates error-free bypass of a bulky DNA lesion. Nucleic Acids Research. 2012; 18:9193-205. [PubMed: 22772988]

Maple JR, Hwang MJ, Stockfisch TP, Dinur U, Waldman M, Ewig CS, Hagler AT. Derivation of class II force fields.1 Methodology and quantum force field for the alkyl functional group and alkane molecules. J Comp Chem. 1994; 15:162-182.

Liu DC, Nocedal J. On the limited memory method for large scale optimization. Mathematics Programming B. 1989; 45:503-528.

Maisuradze CG, Senet P, Czaplewski C, Liwo A, Scheraga HA. Investigation of protein folding by coarse-grained molecular dynamics with the UNRES force field. J Phys Chem A. 2010; 114:44714485. [PubMed: 20166738]

McCammon, JA.; Harvey, SC. Dynamics of Proteins and Nucleic Acids. Cambridge University Press; Cambridge: 1987.

McQuarrie, DA.; Simon, JD. Physical Chemistry: A Molecular Approach. University Science Books; Sausalito, CA: 1997.

Metropolis N, Rosenbluth AW, Rosenbluth MN, Teller AH, Teller E. Equation of state calculations by fast computing machines. J Chem Phys. 1953; 21:1087-1092.

Mlynsky V, Banas P, Walter NG, Sponer J, Otyepka M. QM/MM studies of hairpin ribozyme selfcleavage suggest the feasibility of multiple competing reaction mechanisms. J Phys Chem B. 2011; 115:13911-13924. [PubMed: 22014231]

Nam K, Gao J, York DM. Quantum mechanical/molecular mechanical simulation study of the mechanism of hairpin ribozyme catalysis. J Amer Chem Soc. 2008; 130:4680-4691. [PubMed: 18345664]

Nesterova EN, Federov OU, Poltev VI, Chuprina VP. The study of possible A and B conformations of alternating DNA using a new program for conformational analysis of duplexes (CONAN). J Biomol Struct Dyn. 1997; 14:459-474. [PubMed: 9172646]

Noguti T, Go N. Efficient Monte Carlo method for simulation of fluctuating conformations of native proteins. Biopolymers. 1985; 24:527-546. [PubMed: 3986295] 
Northrup SH, McCammon JA. Simulation methods for protein structure fluctuations. Biopolymers. 1980; 19:1001-1016. [PubMed: 7378544]

Pastor, RW. Techniques and applications of Langevin dynamics simulations. In: Luckhurst, G.; Veracini, C., editors. The Molecular Dynamics of Liquid Crystals. Kluwer Academic Publishers; Amsterdam, The Netherlands: 1994. p. 85-138.

Pearlman DA, Case DA, Caldwell JW, Ross WS, Cheatham TE, Debolt S, Ferguson D, Seibel G, Kollman P. AMBER, a package of computer programs for applying molecular mechanics, normal mode analysis, molecular dynamics and free energy calculations to simulate the structure and energetic properties of molecules. Comp Phys Comm. 1995; 91:1-41.

Perez A, Luque FJ, Orozco M. Dynamics of B-DNA on the microsecond time scale. JACS. 2007; 47:14739-14745.

Perez A, Marchan I, Svozil D, Sponer J, Cheatham TE III, Laughton CA, Orozco M. Refinement of the AMBER force field for nucleic acids: improving the description of $a / \gamma$ conformers. Biophys $J$. 2007; 92:3817-3829. [PubMed: 17351000]

Ponder JW, Wu C, Ren P, Pande VS, Chodera JD, Schnieders MJ, Haque I, Mobley DL, Lanbrecht DS, DiStasio RA Jr, Head-Gordon M, Clark GN, Johnson ME, Head-Gordon T. Current status of the AMOEBA polarizable force field. J Phys Chem B. 114:2549-2564. [PubMed: 20136072]

Repasky MP, Chandrasekhar J, Jorgensen WL. PDDG/PM3 and PDDG/MNDO: Improved semiempirical methods. J Comp Chem. 2002; 23:1601-1622. [PubMed: 12395428]

Reznac J, Fanfrilik J, Salahub D, Hobza P. Semiempirical quantum chemical PM6 method augmented by dispersion and h-bonding correction terms reliably describes various types of noncovalent complexes. J Chem Theory Comput. 2009; 5:1749-1760.

Rick SW, Stuart SJ, Berne BJ. Dynamical fluctuating charge force fields-application to liquid water. J Chem Phys. 1994; 101:6141-6156.

Riley KE, Pitonak M, Jurecka P, Hobza P. Stabilization and structure calculations for noncovalent interactions in extended molecular systems based on wave function and density functional theories. Chem Rev. 2010; 110:5023-5063. [PubMed: 20486691]

Risthaus T, Grimme S. Benchmarking of London Dispersion-Accounting Density Functional Theory Methods on Very Large Molecular Complexes. J Chem Theory Comput. 2013; 9:1580-1591.

Rocha GB, Freire RO, Simas AM, Stewart JJP. RM1: A reparameterization of AM1 for H, C, N, O, P, S, F, Cl, Br, and I. J Comp Chem. 2006; 27:1101-1111. [PubMed: 16691568]

Ryckaert JP, Ciccotti G, Berendsen HJC. Numerical integration of the cartesian equations of motion of a system with constraints: Molecular dynamics of n-alkanes. J Comp Phys. 1977; 23:327-341.

Sauerwine B, Widom M. Kinetic monte carlo method applied to nucleic acid hairpin folding. Phys Review E. 2011; 84:061912.

Saunders M, Houk KN, Wu YD, Still WC, Lipton M, Chong G, Guida WC. Conformations of cycloheptadecane. A comparison of methods for conformational searching. J Am Chem Soc. 1990; 112:1419-1427.

Schlick T. Molecular dynamics-based approaches for enhanced sampling of long-time, large-scale conformational changes in biomolecules. Biology Reports. 2009; 1:51-60. [PubMed: 20948633]

Schlick T, Overton M. A powerful truncated Newton method for potential energy minimization. J Comp Chem. 1987; 8:1025-1039.

Senn HM, Thiel W. QM/MM Methods for Biomolecular Systems. Agnew Chem Int Ed. 2009; 48:1198-1229.

Simmerling C, Miller JL, Kollman PA. Combined locally enhanced sampling and particle mesh Ewald as a strategy to locate the experimental structure of a non-helical nucleicacid. J Am Chem Soc. 1998; 120:7149-7155.

Sindhikara DJ, Kim S, Voter AF, Roitberg AE. Bad seeds sprout perilous dynamics: stochastic thermostat induced trajectory synchronization in biomolecules. J Chem Theory Comput. 2009; 5:1624-1631.

Sgrignani J, Magistrato A. The structural role of Mg2+ ions in a class I RNA polymerase ribozyme: a molecular simulation study. J Phys Chem B. 2012; 116:2259-2268. [PubMed: 22268599] 
Sklenovsky P, Florova P, Banas P, Reblova K, Lankas F, Otyepka M, Sponer J. Understanding RNA flexibility using explicit solvent simulations: the ribosomal and group I intron reverse kink-turn motifs. J Chem Theory Comput. 2011; 7:2963-2980.

Sponer J, Riley KE, Hobza P. Nature and magnitude of aromatic stacking of nucleic acid bases. Phys Chem Chem Phys. 2008; 12:2595-2610. [PubMed: 18464974]

Sponer J, Sponer JE, Petrov AI, Leontis NB. Quantum Chemical Studies of Nucleic Acids: Can We Construct a Bridge to the RNA Structural Biology and Bioinformatics Communities? J Phys Chem B. 2010; 114:15723-15741. [PubMed: 21049899]

Sponer J, Mladek A, Sponer JE, Svozil D, Zgarbova M, Banas P, Jurecka P, Otyepka M. The DNA and RNA sugar-phosphate backbone emerges as the key player. An overview of quantumchemical structural biology and simulation studies. Phys Chem Chem Phys. 2012; 14:1525715277. [PubMed: 23072945]

Stanton RV, Little LR, Merz KM. An examination of a Hartree-Fock molecular mechanical coupled potential. J Phys Chem. 1995; 99:17344-17348.

Steinbach PJ, Brooks BR. Protein simulation below the glass-transition temperature. Dependence on cooling protocol. Chem Phys Lett. 1994; 226:447-452.

Stewart, JJP. Semiempirical molecular orbital methods. In: Lipkowitz, KB.; Boyd, DB., editors. Reviews in Computational Chemistry. VCH; New York: 1990. p. 45-81.

Straub, JE. Optimization techniques with applications to proteins. In: Elber, R., editor. New Developments in Theoretical Studies of Proteins. World Scientific; Singapore: 1996. p. 137-196.

Sugita Y, Okamoto Y. Replica-exchange molecular dynamics method for protein folding. Chem Phys Lett. 1999; 314:141-151.

Swendsen RH, Wang JS. Replica Monte Carlo simulation of spin glasses. Phys Rev Lett. 1986; 57:2607-2609. [PubMed: 10033814]

Szabo, A.; Ostlund, NS. Modern Quantum Chemistry. McGraw-Hill; New York: 1989.

Takada S. Coarse-grained molecular simulations of large biomolecules. Cur Opin Struct Biol. 2012; 22:130-137.

Tan YH, Tan C, Wang J, Luo R. Continuum polarizable force field within the Poisson-Boltzmann framework. J Phys Chem B. 2008; 112:7675-7688. [PubMed: 18507452]

Thiel W. Computational methods for large molecules. J Mol Struct. 1997; 398:1-6.

Tomasi J, Mennucci B, Cammi R. Quantum mechanical continuum solvation models. Chem Rev. 2005; 105:2999-3094. [PubMed: 16092826]

Tuckerman M, Berne BJ, Martyna GJ. Reversible multiple time scale molecular dynamics. J Chem Phys. 1992; 97:1990-2001.

Uberuaga BP, Anghel M, Voter AF. Synchronization of trajectories in canonical molecular-dynamics simulations: observation, explanation and exploitation. J Chem Phys. 2004; 120:6363-6374. [PubMed: 15267525]

Valleau, JP.; Whittington, SG. A Guide to Monte Carlo for Statistical Mechanics: 1. Highways. In: Berne, BJ., editor. Statistical Mechanics A A Modern Theoretical Chemistry. Plenum Press; New York: 1977. p. 137-168.

van Gunsteren, WF.; Berendsen, HJC. Groningen molecular simulation (GROMOS) library manual. BIOMOS; Nijenborgh, Groningen, The Netherlands: 1987.

van Gunsteren WF, Berendsen HJC. Computer simulation of molecular dynamics: Methodology, applications, and perspectives in chemistry. Angew Chem Int Ed Engl. 1990; 29:992-1023.

van Gunsteren WF, Mark AE. Validation of molecular dynamics simulation. J Chem Phys. 1998; 108:6109-6116.

Vorobjev YN, Hermans J. ES/IS: estimation of conformational free energy by combining dynamics simulations with explicit solvent with and implicit solvent continuum model. Biophys Chem. 1999; 78:195-205. [PubMed: 10343388]

Wang J, Cieplak P, Kollman PA. How well does a restrained electrostatic potential (RESP) model perform in calculating conformational energies of organic and biological molecules? J Comp Chem. 2000; 21:1049-1074. 
Wang J, Cieplak P, Li J, Wang J, Cai Q, Hsieh M, Lei H, Luo R, Duan Y. Development of polarizable models for molecular mechanical calculations II: induced dipole models significantly improve accuracy of intermolecular interaction energies. J Phys Chem B. 2011; 115:3100-3011. [PubMed: 21391583]

Wiberg KB. A scheme for strain energy minimization. Application to the cycloalkanes. J Am Chem Soc. 1965; 87:1070-1078.

Winger M, Trzesniak D, Baron R, van Gunsteren WF. On using a too large integration time step in molecular dynamics simulations of coarse-grained molecular models. Phys Chem Chem Phys. 2009; 11:1934-1941. [PubMed: 19280004]

Yang Y, Yu H, York DM, Cui Q. Description of phosphate hydrolysis reactions with the SelfConsistent-Charge Density-Functional-Tight-Binding (SCC-DFTB) theory. 1 Parameterization. J Chem Theory Comp. 2008; 4:2067-2084. 2008.

Young MA, Jayaram B, Beveridge DL. Intrusion of counterions into the spine of hydration in the minor groove of B-DNA: Fractional occupancy of electronegative pockets. J Am Chem Soc. 1997; 119:59-69.

Zerner, MC. Semiempirical molecular orbital methods. In: Lipkowitz, KB.; Boyd, DB., editors. Reviews in Computational Chemistry. VCH; New York: 1991. p. 313-365.

Zgarbova M, Otyepka M, Sponer J, Mladek A, Banas P, Cheatham TE III, Jurecka P. Refinement of the Cornell et al. Nucleic Acids force field based on reference quantum chemical calculations of glycosidic torsion profiles. J Chem Theory Comput. 2011; 7:2886-2902. [PubMed: 21921995]

Zhou Y, Truhlar DG. The M06 suite of density functionals for main group thermochemistry, thermochemical kinetics, noncovalent interactions, excited states, and transition elements: two new functionals and systematic testing of four M06-class functionals and 12 other functionals. Theor Chem Acc. 2008; 120:215-241.

Zhurkin VB, Ulyanov NB, Gorin AA, Jernigan RL. Static and statistical bending of DNA evaluated by Monte Carlo calculations. Proc Natl Acad Sci USA. 1991; 88:7046-7050. [PubMed: 1871119]

Zuo G, Li W, Zhang J, Wang J, Wang W. Folding of a small RNA hairpin based on simulation with replica exchange molecular dynamics. J Phys Chem B. 2010; 114:5835-5839. [PubMed: 20392088] 


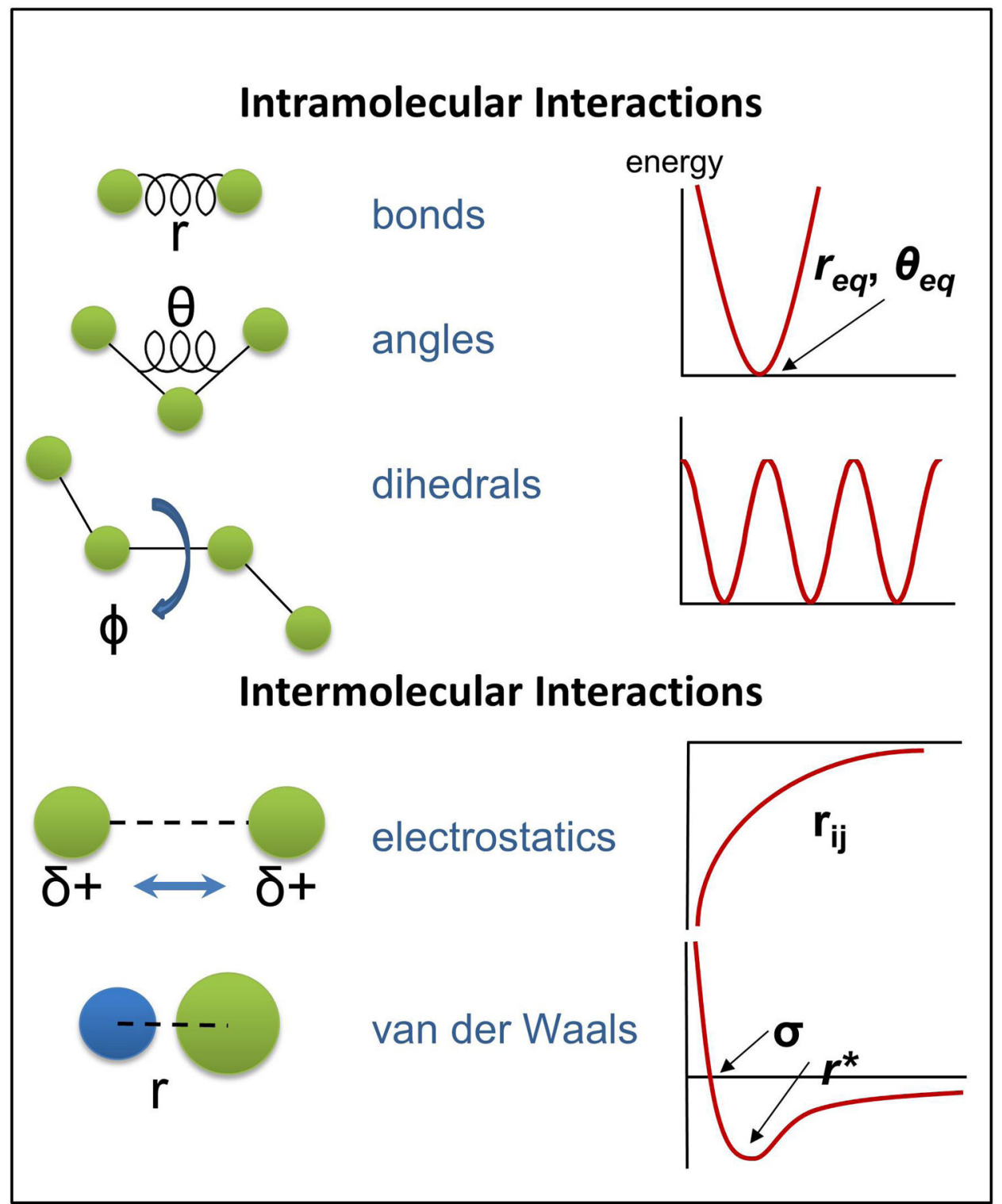

Figure 7.8.1.

Schematic of the interactions in a pairwise additive molecular mechanics force field. 

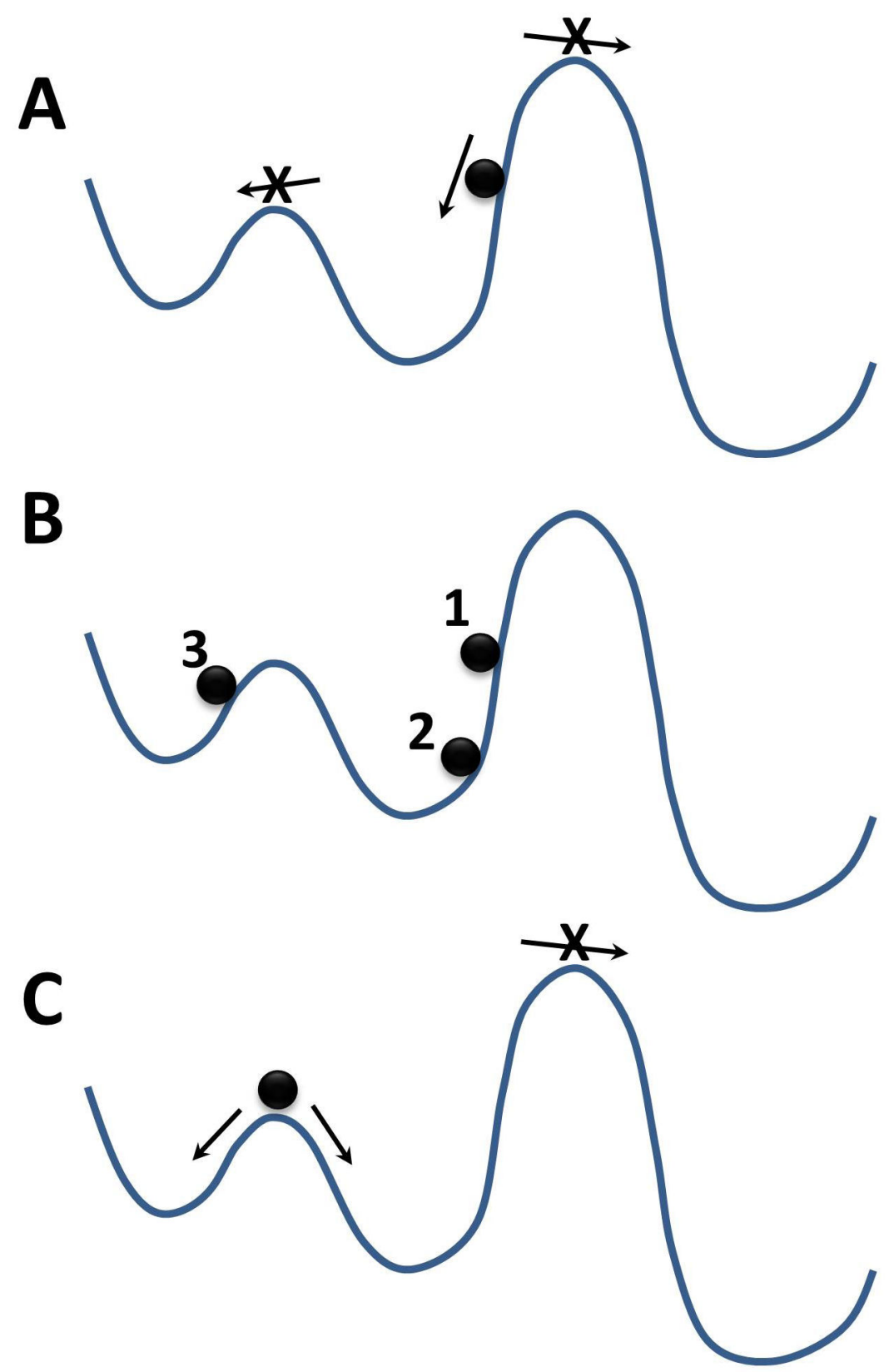

Figure 7.8.2.

Schematic representations of the sampling of various methods. These plots represent the energy of the system along an arbitrary reaction coordinate. The wells represent energy minima in the phase space. The state of the system is depicted by the location of the ball.

(A) Minimization. The system moves to the bottom of the nearest well and barriers are not overcome. (B) Monte Carlo. Each configuration of the system is represented by a number and barrier crossing relates to the move set and total number of moves. (C) Molecular dynamics. The state of the system evolves due to force according to Newton's equations of motion. In short simulations, large barriers will not be surmounted. 